\title{
Low-Loss Broadband Antenna for Efficient Photon Collection from a Coherent Spin in Diamond
}

\author{
D. Riedel, D. Rohner, M. Ganzhorn, T. Kaldewey, P. Appel, E. Neu, R. J. Warburton, and P. Maletinsky* \\ Department of Physics, University of Basel, Klingelbergstrasse 82, Basel CH-4056, Switzerland \\ (Received 31 July 2014; revised manuscript received 4 November 2014; published 30 December 2014)
}

\begin{abstract}
We report the creation of a low-loss broadband optical antenna giving highly directed output from a coherent single spin in the solid state. The device, a crystalline solid-state realization of a dielectric antenna, is engineered for individual nitrogen-vacancy electronic spins in diamond. We demonstrate a directionality close to 10. The photonic structure preserves the high spin coherence of single-crystal diamond $\left(T_{2} \gtrsim 100 \mu \mathrm{s}\right)$. The single-photon count rate approaches a megahertz facilitating efficient spin readout. We thus demonstrate a key enabling technology for quantum applications such as high-sensitivity magnetometry and long-distance spin entanglement.
\end{abstract}

DOI: 10.1103/PhysRevApplied.2.064011

\section{INTRODUCTION}

The electronic spin associated with the nitrogen-vacancy $(\mathrm{NV})$ center in diamond constitutes a versatile quantum system with applications in nanoscale magnetometry [1-3], quantum communication [4-6], and quantum information processing [7]. NV spins in clean single-crystalline diamond exhibit remarkable coherence times up to milliseconds even at room temperature [8] and can be initialized and read out optically [9]. For the majority of applications of NV center spins, the efficiency of collection and detection of the broadband NV photoluminescence (PL) is an essential figure of merit. For example, increased NV PL detection rates lead to improved sensitivities in magnetometry applications [10] and higher two-photon interference rates for entangling remote NV spins [7]. These collection efficiencies, however, are intrinsically limited by the nondirectional emission of NV PL and total internal reflection between the high-index diamond host material and its low-index surrounding.

To overcome these obstacles and to improve the photoncollection efficiency from NV spins, several approaches are currently being pursued. Total internal reflection can be reduced by employing solid immersion lenses (SILs) $[11,12]$ or diamond nanocrystals [13]. Conversely, the directionality of $\mathrm{NV}$ emission can be improved by optical waveguides [14,15], resonators [16,17], or metallic optical antennas $[13,18]$. However, all these approaches suffer from severe drawbacks. SILs and nanocrystals hardly address the directionality of the NV emission, and diamond nanocrystals generally exhibit poor spin-coherence times [10]. Waveguides and resonators operate only in a narrow wavelength range and are, therefore, ill-suited for broadband

*patrick.maletinsky@unibas.ch

Published by the American Physical Society under the terms of the Creative Commons Attribution 3.0 License. Further distribution of this work must maintain attribution to the author(s) and the published article's title, journal citation, and DOI. emitters such as the NV center, and metal-based approaches $[13,18]$ are plagued by high optical losses. Recently, a new alternative approach based on a layered dielectric optical antenna was proposed and demonstrated for individual molecules in a low-index polymer matrix [19]. Such a "dielectric optical antenna" stands out due to its broadband and almost lossless operation, which can, in principle, yield near-unity collection efficiencies for single emitters [20,21]. However, despite their attractiveness, dielectric optical antennas have never been realized for the NV center in diamond where they are particularly well suited: in addition to their excellent performance, they can be manufactured from highquality single-crystal material where the spin coherence is high. The concept is general and versatile: it is potentially powerful for other broadband solid-state emitters.

\section{EXPERIMENT}

In this paper, we present an experimental realization of a dielectric optical antenna for a NV center in diamond. Our approach is both deterministic and reproducible and allows for low-loss broadband operation with a highly directional output, all while preserving the spin coherence of the starting material. Our work promotes the concept of a dielectric optical antenna for quantum emitters in the solid state and, moreover, demonstrates successful antenna operation for a high-index host material, where light extraction is particularly challenging. Our antenna is based on a thin single-crystalline diamond membrane directly bonded to the center of a high-index SIL, as illustrated in Fig. 1(a). The three layers consisting of air (refractive index $n=1.0)$, the diamond film $(n=2.4)$, and the gallium phosphide (GaP, $n=3.3$ ) SIL together form the antenna and lead to the highly directional NV emission. Crucial to the operation of our antenna is the use of a SIL material with a refractive index higher than that of diamond. This index contrast and the thin-diamond membrane are at the heart of the operation of our antenna as they lead to preferential photon emission into the high-index material 



FIG. 1. (a) Schematic of the high-index dielectric antenna structure: a thin-diamond membrane containing NV centers is bonded to a gallium phosphide (GaP) SIL. The majority of the NV PL is emitted within the critical angle $\theta \sim 47^{\circ}$ of the GaP-diamond interface [corresponding to emission within a numerical aperture (NA) of approximately 0.73]. (b) Positioning of the transferred diamond membranes onto the GaP SIL using a micromanipulator. (c) Room-temperature PL spectrum of a single-NV center collected through the dielectric antenna. PL studied in this work passes through a $(675 \pm 50)-\mathrm{nm}$ bandpass filter to improve the signal-to-background ratio.

[22]. The only readily available SIL material with a refractive index significantly larger than 2.4 showing transparency over the $\mathrm{NV}$ emission spectrum is GaP [23], with an index of 3.3. The planar interfaces of airdiamond-GaP form the dielectric antenna. Efficient outcoupling of the photons from the $\mathrm{GaP}$ can be achieved with a hemispherical shape [24]: photons originating from the antenna have normal incidence at the GaP-air interface, preventing total internal reflection [Fig. 1(a)]. We, therefore, employ a hemispherical GaP SIL.

In order to obtain a diamond film of suitable thickness for our antenna, we fabricate diamond membranes (with typical dimensions approximately equal to $20 \mu \mathrm{m} \times$ $20 \mu \mathrm{m} \times 4 \mu \mathrm{m}$ ) from commercially available single-crystal diamond [25]. Exploiting recently developed top-down diamond nanofabrication techniques $[3,15]$, we structure arrays of free-standing membranes with predetermined breaking points [25]. We then detach the membranes using the sharp tip of a micromanipulator and transfer them to the flat surface of the GaP. The GaP SIL has radius $1.00 \mathrm{~mm}$ and is fabricated from bulk material by mechanical polishing. The correctly positioned diamond membranes bond to the SIL surface by van der Waals forces [Fig. 1(b)]. As a last step, we adjust the thicknesses $d$ of the diamond layer by further thinning of the bonded membranes by successive reactive ion etching steps [25].

\section{RESULTS AND DISCUSSION}

The PL emission from optical dipoles embedded in dielectric antennas exhibits characteristic radiation patterns
[19], signatures of the antenna effects we wish to demonstrate and exploit. We record these radiation patterns for native NV centers in our antenna structures through back focal plane (BFP) imaging. The NV PL is excited using a pulsed tunable laser source (NKT EXW-12, $\lambda=560 \mathrm{~nm}$, repetition rate $78 \mathrm{MHz}$ ) and detected using a narrow-band filter to isolate the strongly wavelength-dependent interference features. As a first step, we image the PL of NV ensembles in antennas with $d=815 \mathrm{~nm}$ and $d=560 \mathrm{~nm}$ [Figs. 2(a) and 2(c)]. We observe a radiation pattern consisting of multiple interference rings whose positions strongly vary with $d$. For all our BFP images, the NV PL is confined to within a maximal emission angle $\theta_{c, 1}$, which corresponds closely to the critical angle at the diamond$\mathrm{GaP}$ interface (approximately $47^{\circ}$ ). This observation demonstrates highly directional NV emission into a NA of approximately 0.73 . Importantly, this value is significantly smaller than the NA of 0.8 (corresponding to $\theta_{\mathrm{obj}}=53^{\circ}$ ) of the microscope objective we employ. In addition, we observe a region of relatively low PL intensity bounded
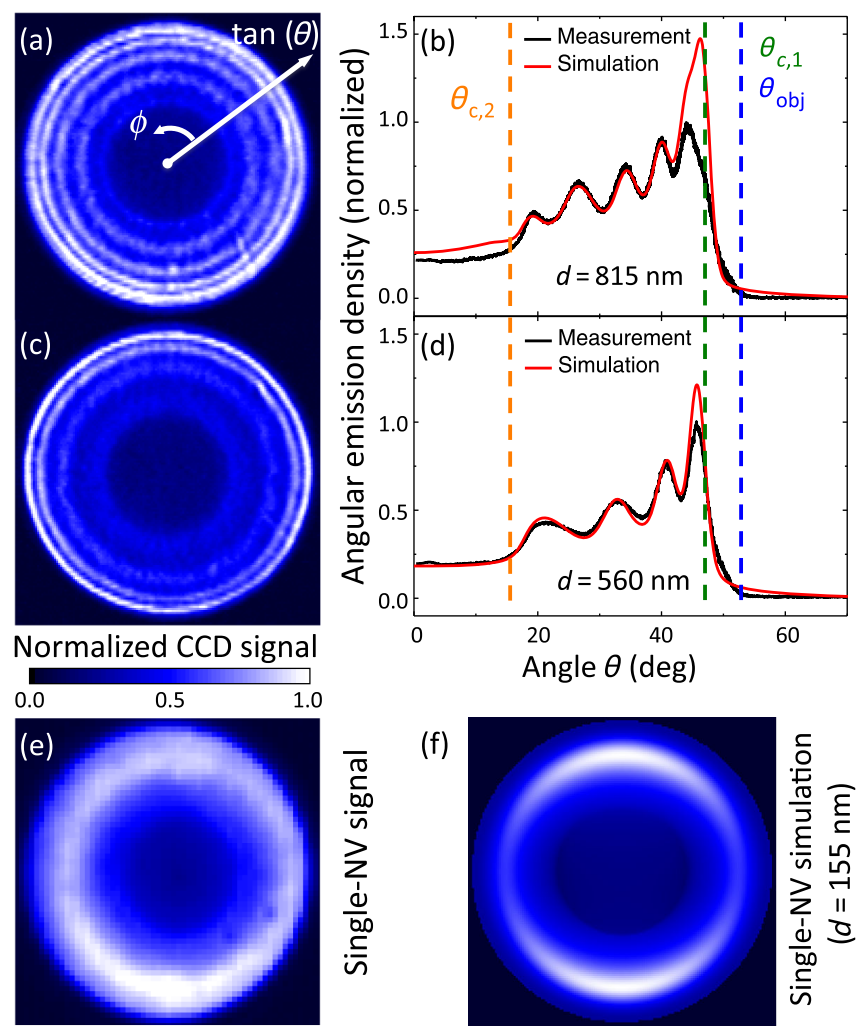

FIG. 2. Angular radiation patterns of NV ensembles in the dielectric antenna obtained by back focal plane (BFP) imaging for (a) $d=815 \mathrm{~nm}$ and (c) $d=560 \mathrm{~nm}$. (b),(d) Average of the measured BFP images over azimuthal angle $\phi$ and calculated emission patterns. (e) Broadband emission pattern of a single-NV center. (f) Calculated BFP image for a NV center in a diamond membrane with $d=155 \mathrm{~nm}$ and $h=140 \mathrm{~nm}$. The color bar applies to all images in the figure. (a)-(d) use a $(680 \pm 5)-\mathrm{nm}$ bandpass filter; (e) a (675 \pm 50$)$-nm bandpass filter. 
by an angle $\theta_{c, 2}$ in the center of each BFP image. This region corresponds to $\mathrm{PL}$ light escaping the antenna through the diamond-air interface. Because of the collection through the GaP material, the observed value, thus, corresponds to the critical angle between $\mathrm{GaP}$ and air, $\theta_{c, 2} \sim 18^{\circ}$.

For a detailed analysis of the radiation patterns, we average the measured BFP images over the azimuthal angle $\phi$ and compare the resulting emission profile to an analytical calculation [Figs. 2(b) and 2(d)]. These calculations rely on established procedures for the analysis of the far-field emission from layered dielectric structures [22,26,27] (for details, see Ref. [25]). We use tabulated values for refractive indices of diamond and $\mathrm{GaP}$ [28] and account for the NV PL spectrum by introducing wavelength-dependent weighting factors for the emission profile, which we discretize in steps of $5 \mathrm{~nm}$. For both values of $d$, we detect PL from a large ensemble of NVs in the diamond membrane and we assume these NVs to be homogeneously distributed and randomly oriented. With the exception of the highest emission angles $\theta \sim \theta_{\text {obj }}$, our experimental results show excellent agreement with the theoretical expectations. In particular, the characteristic oscillations we observe in Figs. 2(b) and 2(d) (and which persist despite our ensemble average over dipole orientation and position) allow us to determine precisely the only free parameter in our fit, the thickness $d$ of the diamond layer, which we find to be in good agreement with independent measurements [25].

Upon further thinning of the diamond membrane, the areal density of NV centers gradually decreases, until eventually, for a thickness of $d \lesssim 150 \mathrm{~nm}$, we are able to observe NV emission from isolated spots [Fig. 3(a)]. Figure 2(e) displays the angular radiation pattern of the broadband PL of such a spot. This emission pattern agrees well with the calculated BFP image for a single-NV center [Fig. 2(f)] for which we take into account the two optical dipoles responsible for NV emission [29] and use $d$ and $h$ (the distance between the NV center and diamond$\mathrm{GaP}$ interface) as fit parameters. As a result of the twodimensional NV dipole moment, we expect only weakly polarized antenna emission and, therefore, perform unpolarized detection of our BFP images.

To substantiate further that our observed isolated emission spots indeed stem from single NVs, we determine the photon autocorrelation function $g^{(2)}(t)$ of the detected PL. Figure 3(b) shows $g^{(2)}(t)$ measured on NV no. 1. The data exhibit strong photon antibunching with $g^{(2)}(0) \lesssim 0.1$ and a decay of the pulsed PL on a time scale $\tau=(15.4 \pm 0.2)$ ns. The decay time is a typical NV center fluorescence lifetime: this value, therefore, shows that our antenna fabrication process does not induce unwanted nonradiative decay channels for the NV.

To study the directionality $\eta$ of single-photon emission from our antenna, we compare PL emission rates detected
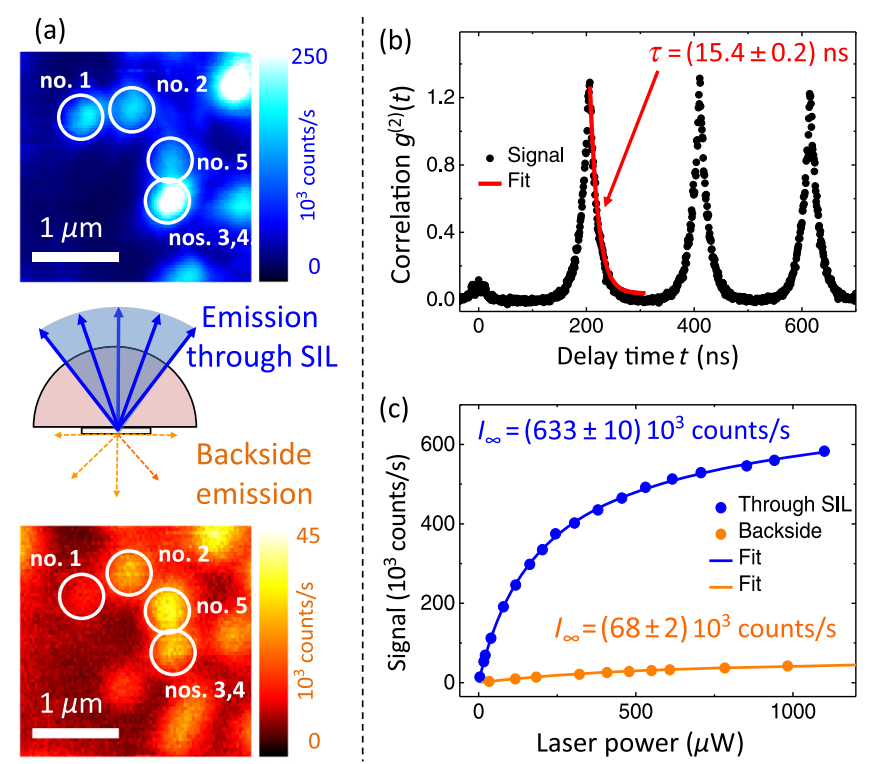

FIG. 3. (a) PL images of individual NV centers recorded through the SIL and through the backside of the antenna. (b) Photon autocorrelation measurement $g^{(2)}(t)$ of $\mathrm{NV}$ center no. 1 using pulsed excitation. The data show $g^{(2)}(0) \lesssim 0.1$ and an exponential PL decay with a lifetime $\tau=(15.4 \pm 0.2) \mathrm{ns}$. (c) Comparison of PL saturation of a single NV in the dielectric antenna when detecting through the GaP SIL and through the backside of the antenna. NV PL is detected using a $(675 \pm 50)$-nm bandpass filter.

through the SIL and through the backside of the antenna [upper and lower panels in Fig. 3(a), respectively]. Our confocal scans reveal several single NVs (labeled as nos. 1-5 in the figure) scattered throughout the diamond membrane. The random positioning of the native NVs in our structure allows us to observe the effect of varying vertical positions $h$ on antenna performance: NVs which are well coupled to the antenna (nos. 1, 3, and 4) appear bright when observed through the SIL and dark through the backside, while less-well-coupled NVs (no. 5) exhibit the opposite behavior.

For a more quantitative analysis of the antenna directionality, we compare the saturated PL count rate $I_{\infty}$ of the well-coupled NV no. 1 measured through the GaP SIL and from the antenna backside. We obtain $I_{\infty}$ by fitting the measured PL saturation curves [Fig. 3(c)] with $I(P)=$ $I_{\infty}\left(1+P / P_{\text {sat }}\right)^{-1}+b P$ [4], where $P$ is the laser power, $P_{\text {sat }}$ the saturation power, and $b P$ accounts for background fluorescence. For PL detection through the SIL, we find $I_{\infty, \mathrm{SIL}}=(633 \pm 10) \times 10^{3}$ counts $/ \mathrm{s}$, while we obtain $I_{\infty, \mathrm{BS}}=(68 \pm 2) \times 10^{3}$ counts $/ \mathrm{s}$ for backside detection [30]. This corresponds to an enhancement of the detection rate by the antenna, which we directly relate to the directionality of our antenna; i.e., we find $\eta=9.3$.

We note that the directionality is an underestimation of the directionality of the dielectric antenna itself, as approximately $45 \%$ of the NV emission is lost on outcoupling, 
approximately $30 \%$ by reflection at the GaP-air interface (a consequence of the abrupt change in reflective index), and another approximately $10 \%$ by scattering losses (a consequence of imperfections in the GaP material [25]). We also note that a possible source of systematic error is the fact that our current realization of our antenna demands slightly different experimental conditions for top- and bottom-side collection. Specifically, top-side collection is performed in a nonconfocal imaging mode since the emission pattern of our antenna has poor overlap with the Gaussian mode of the single-mode fiber, which represents the "pinhole" in our microscope. Backside collection, however, is performed with confocal detection via a singlemode fiber since the high density of NVs in our antenna did not allow us to isolate single NVs otherwise. However, we expect backside confocal detection to be very efficient [25] such that the systematic error is small. We emphasize that $\eta=9.3$ represents the performance of the entire antenna device without correcting for known losses at the GaP-air interface. For the $d$ and $h$ as determined from the radiation pattern for NV no. 1, the NA of the objective lens, and including reflection losses at the GaP-air interface, we calculate a directionality of $\eta=13.9$, slightly higher than the measured value.

With single-NV centers isolated in our dielectric antenna, we now turn our attention to their electronic spin properties. To that end, we perform optically detected magnetic resonance (ODMR) [9] on individual NVs in our antenna. The ODMR resonance frequencies are determined by the strength of an external magnetic field $\vec{B}$ and the orientation between the NV axis and $\vec{B}$. We apply $\vec{B}$ in an oblique direction with respect to the four possible NV



FIG. 4. (a) Optically detected magnetic resonance (ODMR) of the NVs highlighted in Fig. 3(a) $(|\vec{B}| \sim 4 \mathrm{mT})$, each exhibiting a different orientation within the diamond lattice. The low ODMR contrast results from the low microwave power at the NV location. (b) ODMR amplitude of NV no. 1 imaged in the back focal plane. (c) Hahn spin-echo measurement on $\mathrm{NV}$ no. 4 yielding $T_{2}=(114 \pm 9) \mu \mathrm{s}(|\vec{B}| \sim 11.5 \mathrm{mT})$. axes such that ODMR allows us to differentiate NVs with different orientations. Figure 4(a) shows the ODMR for NV nos. 1-4 in a static magnetic field. The ODMR enables us to probe the spin dependence of the BFP image in Fig. 2(e). We record the difference of PL in the BFP when driving the NV on and off spin resonance. The corresponding spin-resolved BFP image is shown in Fig. 4(b) and is, up to a constant scaling factor, identical to Fig. 2(e). In particular, the ODMR contrast is constant throughout the BFP, and no ODMR contrast is detected in the BFP when we drive the spin resonance of one of the NV no. 1's neighbors (all of which have orientations different from NV no. 1).

Finally, we use coherent manipulation of the NVs' electronic spins to probe their $T_{2}$ times (strictly, the Hahn-echo coherence times). Figure 4(c) shows a Hahnecho measurement performed on NV no. 4. We find $T_{2}=(114 \pm 9) \mu \mathrm{s}$, which is typical for the chemical vapor deposition diamond material we employ here. This demonstrates that fabrication of the dielectric membrane preserves the long NV electronic spin-coherence times.

The radical change in the radiation patterns and the highly directed PL emission from single NVs demonstrate proper operation of the dielectric antenna. Still, several factors can be improved in future implementations. The losses at the GaP-air interface can be avoided primarily by an antireflection coating but also by smoother surfaces; the losses at large angles need to be investigated and mitigated. The emission mode of the antenna can be further improved by using (111)-oriented diamond where the NV emission dipoles show an orientation better suited for our antenna [15]. Lastly, a slight improvement can be gained by optimization of $d$ and $h$ (by creating NVs at well-defined depths through ion implantation or $\delta$ doping [31]): according to our calculations, the fraction of PL coupled into the GaP SIL can be increased from the present $95 \%(d=$ $155 \mathrm{~nm}, h=140 \mathrm{~nm}$ ) to above $97.5 \%$ [25]. We note also that using an optimized background filter will readily reduce the losses in our setup, which are presently $\gtrsim 25 \%$ [Fig. 1(c)]. Altogether, an improvement in NV PL detection efficiency by more than one order of magnitude can be envisaged.

\section{SUMMARY}

In summary, we apply the powerful concept of a dielectric optical antenna [19] to single quantum emitters in the solid state. We demonstrate successful antenna operation and the addressing of single-NV spins in the antenna. The antenna has broadband operation, and it preserves the advantageous optical and spin properties of the single-crystal diamond starting material. With the high antenna directionality, photon-collection efficiency, and spin-coherence times, we are able to demonstrate simultaneously that our results present significant advances over previous approaches in the field of diamond nanophotonics 
[11-18]. In particular, the dielectric antenna constitutes the only demonstrated approach, which, in principle, allows for near-unity collection efficiency [20] for NVs. Further improvements to our structure should, therefore, allow for record-high single-NV count rates approaching $10 \mathrm{MHz}$. Our antenna design is immediately applicable to a variety of applications ranging from wide-field magnetic imaging [32] to nanoscale nuclear spin detection [33] and entanglement of remote spins [7], where, in all cases, it is poised to lead to a significant leap in performance. Finally, we emphasize that our approach is not limited to NV centers - other color centers in diamond [34] can also profit from the antenna-or even to diamond itself: silicon carbide [35] and other solid-state hosts can be integrated into planar dielectric antennas.

\section{ACKNOWLEDGMENTS}

We thank M. Kasperczik and L. Novotny for help with our back focal plane imaging experiments. We gratefully acknowledge financial support from SNI, NCCR QSIT, EU FP7 ITN S ${ }^{3}$ NANO, SNSF Grants No. 200021_143697 and No. 200021_132313. This research has been partially funded by the European Commission's 7 Framework Program (FP7/2007-2013) under Grant Agreement No. 611143 (DIADEMS).

D. Riedel and D. Rohner contributed equally to this work.

[1] J. R. Maze, P. L. Stanwix, J. S. Hodges, S. Hong, J. M. Taylor, P. Cappellaro, L. Jiang, M. V. G. Dutt, E. Togan, A. S. Zibrov, A. Yacoby, R. L. Walsworth, and M. D. Lukin, Nanoscale magnetic sensing with an individual electronic spin in diamond, Nature (London) 455, 644 (2008).

[2] G. Balasubramanian, I. Y. Chan, R. Kolesov, M. Al-Hmoud, J. Tisler, C. Shin, C. Kim, A. Wojcik, P. R. Hemmer, A. Krueger, T. Hanke, A. Leitenstorfer, R. Bratschitsch, F. Jelezko, and J. Wrachtrup, Nanoscale imaging magnetometry with diamond spins under ambient conditions, Nature (London) 455, 648 (2008).

[3] P. Maletinsky, S. Hong, M. S. Grinolds, B. J. M. Hausmann, M. D. Lukin, R. L. Walsworth, M. Loncar, and A. Yacoby, A robust scanning diamond sensor for nanoscale imaging with single nitrogen-vacancy centres, Nat. Nanotechnol. 7, 320 (2012).

[4] C. Kurtsiefer, S. Mayer, P. Zarda, and H. Weinfurter, Stable solid-state source of single photons, Phys. Rev. Lett. 85, 290 (2000).

[5] R. Brouri, A. Beveratos, J. P. Poizat, and P. Grangier, Photon antibunching in the fluorescence of individual color centers in diamond, Opt. Lett. 25, 1294 (2000).

[6] A. Beveratos, R. Brouri, T. Gacoin, A. Villing, J. P. Poizat, and P. Grangier, Single photon quantum cryptography, Phys. Rev. Lett. 89, 187901 (2002).
[7] H. Bernien, B. Hensen, W. Pfaff, G. Koolstra, M. S. Blok, L. Robledo, T. H. Taminiau, M. Markham, D. Twitchen, L. Childress, and R. Hanson, Heralded entanglement between solid-state qubits separated by three metres, Nature (London) 497, 86 (2013).

[8] G. Balasubramanian, P. Neumann, D. Twitchen, M. Markham, R. Kolesov, N. Mizuochi, J. Isoya, J. Achard, J. Beck, J. Tissler, V. Jacques, P. R. Hemmer, F. Jelezko, and J. Wrachtrup, Ultralong spin coherence time in isotopically engineered diamond, Nat. Mater. 8, 383 (2009).

[9] A. Gruber, A. Dräbenstedt, C. Tietz, L. Fleury, J. Wrachtrup, and C. von Borczyskowski, Scanning confocal optical microscopy and magnetic resonance on single defect centers, Science 276, 2012 (1997).

[10] L. Rondin, J. P. Tetienne, T. Hingant, J. F. Roch, P. Maletinsky, and V. Jacques, Magnetometry with nitrogenvacancy defects in diamond, Rep. Prog. Phys. 77, 056503 (2014).

[11] P. Siyushev, F. Kaiser, V. Jacques, I. Gerhardt, S. Bischof, H. Fedder, J. Dodson, M. Markham, D. Twitchen, F. Jelezko, and J. Wrachtrup, Monolithic diamond optics for single photon detection, Appl. Phys. Lett. 97, 241902 (2010).

[12] L. Marseglia, J. P. Hadden, A. C. Stanley-Clarke, J. P. Harrison, B. Patton, Y. L. D. Ho, B. Naydenov, F. Jelezko, J. Meijer, P. R. Dolan, J. M. Smith, J. G. Rarity, and J. L. O'Brien, Nanofabricated solid immersion lenses registered to single emitters in diamond, Appl. Phys. Lett. 98, 133107 (2011).

[13] J. Wolters, G. Kewes, A. W. Schell, N. Nüsse, M. Schoengen, B. Löchel, T. Hanke, R. Bratschitsch, A. Leitenstorfer, T. Aichele, and O. Benson, Coupling of single nitrogenvacancy defect centers in diamond nanocrystals to optical antennas and photonic crystal cavities, Phys. Status Solidi (b) 249, 918 (2012).

[14] T. M. Babinec, B. J. M. Hausmann, M. Khan, Y. Zhang, J. R. Maze, P. R. Hemmer, and M. Loncar, A diamond nanowire single-photon source, Nat. Nanotechnol. 5, 195 (2010).

[15] E. Neu, P. Appel, M. Ganzhorn, J. Miguel-Sánchez, M. Lesik, V. Mille, V. Jacques, A. Tallaire, J. Achard, and P. Maletinsky, Photonic nano-structures on (111)oriented diamond, Appl. Phys. Lett. 104, 153108 (2014).

[16] B. J. M. Hausmann, B. Shields, Q. Quan, P. Maletinsky, M. McCutcheon, J. T. Choy, T. M. Babinec, A. Kubanek, A. Yacoby, M. D. Lukin, and M. Loncar, Integrated diamond networks for quantum nanophotonics, Nano Lett. 12, 1578 (2012).

[17] A. Faraon, P. E. Barclay, C. Santori, K. M. C. Fu, and R. G. Beausoleil, Resonant enhancement of the zero-phonon emission from a colour centre in a diamond cavity, Nat. Photonics 5, 301 (2011).

[18] J. T. Choy, B. J. M. Hausmann, T. M. Babinec, I. Bulu, M. Khan, P. Maletinsky, A. Yacoby, and M. Lončar, Enhanced single-photon emission from a diamond-silver aperture, Nat. Photonics 5, 738 (2011).

[19] K. G. Lee, X. W. Chen, H. Eghlidi, P. Kukura, R. Lettow, A. Renn, V. Sandoghdar, and S. Götzinger, A planar dielectric antenna for directional single-photon emission 
and near-unity collection efficiency, Nat. Photonics 5, 166 (2011).

[20] X. W. Chen, S. Götzinger, and V. Sandoghdar, 99\% efficiency in collecting photons from a single emitter, Opt. Lett. 36, 3545 (2011).

[21] X.-L. Chu, T. J. K. Brenner, X.-W. Chen, Y. Ghosh, J. A. Hollingsworth, V. Sandoghdar, and S. Götzinger, Experimental realization of an optical antenna designed for collecting $99 \%$ of photons from a quantum emitter, Optica 1, 203 (2014).

[22] L. Luan, P. R. Sievert, B. Watkins, W. Mu, Z. Hong, and J. B. Ketterson, Angular radiation pattern of electric dipoles embedded in a thin film in the vicinity of a dielectric half space, Appl. Phys. Lett. 89, 031119 (2006).

[23] K. M. C. Fu, C. Santori, P. E. Barclay, I. Aharonovich, S. Prawer, N. Meyer, A. M. Holm, and R. G. Beausoleil, Coupling of nitrogen-vacancy centers in diamond to a GaP waveguide, Appl. Phys. Lett. 93, 234107 (2008).

[24] Q. Wu, G. D. Feke, R. D. Grober, and L. P. Ghislain, Realization of numerical aperture 2.0 using a gallium phosphide solid immersion lens, Appl. Phys. Lett. 75, 4064 (1999).

[25] See Supplemental Material at http://link.aps.org/ supplemental/10.1103/PhysRevApplied.2.064011 for details about employed materials, fabrication processes and analytical calculations of the antenna emission patterns.

[26] J. Y. Courtois, J. M. Courty, and J. C. Mertz, Internal dynamics of multilevel atoms near a vacuum-dielectric interface, Phys. Rev. A 53, 1862 (1996).

[27] M. A. Lieb, J. M. Zavislan, and L. Novotny, Singlemolecule orientations determined by direct emission pattern imaging, J. Opt. Soc. Am. B 21, 1210 (2004).

[28] M. Bass, C. DeCusatis, J. M. Enoch, V. Lakshminarayanan, G. Li, C. MacDonald, V. N. Mahajan, and E. V. Stryland,
Handbook of Optics, 3rd ed. (McGraw-Hill, New York, 2009), Vol. IV.

[29] T. P. M. Alegre, C. Santori, G. Medeiros-Ribeiro, and R. G. Beausoleil, Polarization-selective excitation of nitrogen vacancy centers in diamond, Phys. Rev. B 76, 165205 (2007).

[30] We note that this value of $I_{\infty}$ is similar but slightly smaller to the value we find for single NVs measured in unstructured bulk diamond in our setup.

[31] K. Ohno, F. J. Heremans, L. C. Bassett, B. A. Myers, D. M. Toyli, A. C. Bleszynski Jayich, C. J. Palmstrom, and D. D. Awschalom, Engineering shallow spins in diamond with nitrogen delta-doping, Appl. Phys. Lett. 101, 082413 (2012).

[32] D. Le Sage, K. Arai, D. R. Glenn, S. J. DeVience, L. M. Pham, L. Rahn-Lee, M. D. Lukin, A. Yacoby, A. Komeili, and R.L. Walsworth, Optical magnetic imaging of living cells, Nature (London) 496, 486 (2013).

[33] T. Staudacher, F. Shi, S. Pezzagna, J. Meijer, J. Du, C. A. Meriles, F. Reinhard, and J. Wrachtrup, Nuclear magnetic resonance spectroscopy on a (5-nanometer)3 sample volume, Science 339, 561 (2013).

[34] E. Neu, D. Steinmetz, J. Riedrich-Möller, S. Gsell, M. Fischer, M. Schreck, and C. Becher, Single photon emission from silicon-vacancy colour centres in chemical vapour deposition nano-diamonds on iridium, New J. Phys. 13, 025012 (2011).

[35] D. Riedel, F. Fuchs, H. Kraus, S. Väth, A. Sperlich, V. Dyakonov, A. A. Soltamova, P. G. Baranov, V. A. Ilyin, and G. V. Astakhov, Resonant addressing and manipulation of silicon vacancy qubits in silicon carbide, Phys. Rev. Lett. 109, 226402 (2012). 